\title{
U.PH.O AND MAGO: TWO USEFUL INSTRUMENTS IN SUPPORT OF PHOTOGRAMMETRIC UAV SURVEY
}

\author{
S. Gagliolo, D. Passoni, B. Federici, I. Ferrando, D. Sguerso
}

University of Genoa, DICCA - Laboratory of Geodesy, Geomatics and GIS, Via Montallegro 1, 16145 Genoa

(sara.gagliolo, ilaria.ferrando)@edu.unige.it, (bianca.federici, domenico.sguerso)@unige.it, daniele.passoni@dicca.unige.it

KEY WORDS: UAV, Planning, Point cloud, Orthophoto, Emergency, Precision

\begin{abstract}
:
In emergency and critical scenarios, the UAV could play a key role in accessing unreachable sites in a safe and rapid way, guaranteeing at the same time the necessary accuracy and precision of the survey. In this context, UAV survey campaigns have been performed by the authors in Norcia (Italy), hit by tragic seismic events in August and October 2016. The surveys were motivated by the artistic and historical value of monuments, the need to plan and design the restoring and retrofitting of buildings, and also to quantify and manage the ruins. Goal of such surveys was the description of the structures geometry with a centimetric precision and a high level of reliability. Recently, the authors have conceived two tools, U.Ph.O (Unmanned Photogrammetric Office) and MAGO (Adaptive Mesh for Orthophoto Generation), dedicated to the planning and restitution phases of the survey, respectively. U.Ph.O. and MAGO are here applied to two different buildings in Norcia, i.e. the Civic Tower of Norcia and San Salvatore Church of Campi di Norcia. The former is a standing-out structure, surrounded by the complex of the historical centre, while the latter is located in an isolated site in the countryside. These features make the survey planning and the orthophoto reconstruction completely different, mainly due to the different optimal shooting geometry and the presence or absence of obstructions.
\end{abstract}

\section{INTRODUCTION}

The Unmanned Aerial Vehicles (UAVs) are widely used in many different fields: video and photo documentation, security and monitoring, surveillance and reconnaissance, inspection, cultural heritage and archaeological survey, precision agriculture and cadastral applications (Colomina and Molina, 2014; Everaerts, 2008; Remondino et al., 2011; Sona et al., 2016). In emergency scenarios, such as in case of a natural or anthropogenic disaster, UAVs could play a key role in accessing unreachable sites in a safe and rapid way, guaranteeing at the same time the necessary accuracy and precision of the survey (Achille et al., 2015; Adams et al., 2011; Ballarin et al., 2013; Gagliolo et al, 2017; Meyer et al, 2015).

The achievement of metrically reliable final products is still an outstanding topic for photogrammetric purposes (Cryderman et al., 2014; Grün, 1980; James et al., 2017; Torlegård, 1980), and it can not disregard accurate planning and post-processing phases.

The planning is typically managed using dedicated tools, adapted from the traditional design to UAV survey technique. However, UAV operating modes substantially differ from the model of traditional aerial photogrammetry, which consists of parallel camera axes, orthogonal to the base between two cameras. Indeed, UAV survey is characterized by tilted shooting geometry, due to the flight instability, which affects UAVs more than other aircrafts. Hence, a higher image overlapping is requested during UAV survey with respect to aerial photogrammetry.

In this context, a new tool for UAV photogrammetric survey planning, called U.Ph.O. (Unmanned Photogrammetric Office), has been implemented in MATLAB ${ }^{\circ}$ environment (Passoni et al., 2018). U.Ph.O. is intended to account for the morphology of the surveyed object and the presence of occlusions in the a-priori estimation of the reachable metric precision. To pursue this goal, U.Ph.O. is based on a least squares rigorous approach of network simulation, that requires as inputs the relative flight height, the longitudinal and transversal image overlapping, the internal orientation parameters, the shooting geometry, the GCPs (Ground Control Points) coordinates, and an even coarse DSM (Digital Surface Model) representing the scene. The shooting points coordinates are estimated by U.Ph.O. itself in a preprocessing phase. The output products are the realistic estimates of images overlapping and of expected precisions of the survey. Also the acquisition and the elaboration methods to generate detailed 3D products, e.g. point clouds, meshes and DSMs, are improving in the last years. In parallel, 2D products, i.e. planimetry, sections and orthophotos, are still used. In this scenario, orthophotos are widely employed to perform highprecision measurements and to better interpret the thematic description of the surveyed area. They are realized starting from a DSM (traditional approach) or a mesh (photogrammetric software approach). The achievement of the highest possible resolution (in the order of the GSD, Ground Sample Distance) and the approximation introduced by the use of a 3D model are key issues for the $2 \mathrm{D}$ products generation.

A tool, called MAGO (Adaptive Mesh for Orthophoto Generation), has been implemented in $\mathrm{C}++$ environment to generate orthophoto (and eventually the DSM), starting from a point cloud representing the object, IO and EO (Internal and External Orientation) parameters (Gagliolo et al., 2019). To produce the orthophoto, MAGO employs a step-by-step adaptive mesh, generated considering a specific triangular plain area as intersection between the point cloud and the collinearity ray. This strategy allows to limit approximation and loss of definition due to a coarse mesh reconstruction.

U.Ph.O. and MAGO were applied to achieve planning and restitution phases of the survey campaign in Norcia (Italy) performed by the authors after the tragic seismic events in August and October 2016. The surveys were motivated by the artistic and historical value of monuments, the need to plan and design the restoring and retrofitting of buildings, and also to quantify and manage the ruins. Goal of such surveys was the description of the structures geometry with a centimetric precision and a high level of reliability. In particular, the case studies of the Civic Tower in 
Norcia and San Salvatore Church in Campi di Norcia are here analysed.

Agisoft PhotoScan`( (2019) was used to produce some input data of U.Ph.O and MAGO, i.e. images orientation and point cloud reconstruction.

The present dissertation is organized as follows: U.Ph.O. and MAGO workflows are described in sections 2 and 3 , respectively; the application on the considered case studies is presented in section 4 ; the preliminary results are given and commented in section 5; the final considerations conclude the paper in the last section.

\section{U.PH.O. WORKFLOW}

The aim of U.Ph.O. (Unmanned Photogrammetric Office) is to provide the theoretical instruments to make a reliable and complete survey planning with an evaluation of the expected precisions.

The required input data to compute the achievable precisions are:

1. shooting geometry of the camera, potentially changing along the UAV route;

2. relative flight height and/or the GSD;

3. longitudinal and transversal images overlapping on the UAV navigation route;

4. DSM (even coarse) to describe the object of the survey.

The U.Ph.O. workflow begins with the input of camera IO parameters (principal point coordinates $\left(x_{0}, y_{0}\right)$, focal length $c$, and sensor size). Then, the user identifies the area of interest with a Graphical User Interface (GUI), where performing a polygonal selection over a background layer. Hence, the planning constraints (camera model, nadiral or oblique shooting geometry, longitudinal and transversal images overlapping, strips directions, and flight altitude) are requested for a preliminary survey planning. The flight is planned according to the input parameters and the strips are designed at constant altitude. These choices are usual in most planning software, because they represent the most conservative solution to limit the batteries consumption and, therefore, to guarantee a safer flight. The outputs of this first phase of planning are the shooting points coordinates.

Hence, the so-defined flight parameters can be checked, to verify if they satisfy the precision requirements of the survey. The takeoff point coordinates and a DSM of the study area are requested in order to obtain a realistic description of the visibility and of the effective overlapping of images. The output are 2D raster maps, i.e. the visibility map and the occurence map, as explained in the following.

The DSM is particularly important to take into account the obstructions caused by anthropization and vegetation in the study area. In general, a resolution of about $5 \mathrm{~m}$ is appropriate for territorial surveys. In case a DSM is not available or not updated, e.g. as a result of a natural disaster, an exploratory survey to produce a DSM can be performed at a higher altitude than the planned one. This allows to cover the area with few images, avoiding obstacles to the flight and speeding-up the processing to obtain an even rough DSM, thanks to the largely efficient surface reconstruction algorithms, typically present in most photogrammetric software.

To produce the visibility map, U.Ph.O. checks which DSM cells are visible from each image projection centre. According to the authors experience, there are no commercial software which provide this information, although it is crucial to estimate both the number of collinearity rays per object point and the overlap between images and adjacent strips. This check is highly relevant in case of a flight over a mountainous area or around a structure or an urban environment, suggesting changes to the planned flight to meet the survey requirements. Thanks to the visibility analysis, the DSM cells are classified as visible or occluded areas. Then, the collinearity principles are used to count how many times a DSM cell appears on the images, to produce the occurrence map.

The last phase consists in the estimation of the expected precisions through the rigorous method implemented in U.Ph.O. The number and the positions of GCPs are taken into account, acting as block constraints; hence, their presence and arrangement could substantially modify the expected precision estimation. The coordinates of GCPs are associated to their standard deviation; the default value is set to $3 \mathrm{~cm}$, supposing a GNSS (Global Navigation Satellite System) survey, and it could be modified according to the actual survey method of GCPs coordinates.

The rigorous method implemented in U.Ph.O. is applied to each DSM cell independently. For each cell, a block design matrix $A$ is computed from the corresponding occurrence map pixel and the survey parameters (position and attitude of every camera). $A$ is a $m \times 3$ matrix, where $m$ is equal to twice the times the cell is seen in the images. The inversion of the normal matrix $N$ allows to build the covariance matrix $C_{x x}$, which provides the planimetric and altimetric standard deviations of each DSM cell. The visibility, occurrence and precision maps are computed cell by cell with the same resolution of DSM.

The whole U.Ph.O. workflow is iterative. In facts, if the expected precisions do not satisfy the user requirements, or the overlapping is not adequate, or too many points lie into obstructed areas, it is possible to modify the planning parameters, with particular attention to the flight altitude and the overlapping, and to process the project again.

U.Ph.O. workflow is graphically represented in Figure 1.

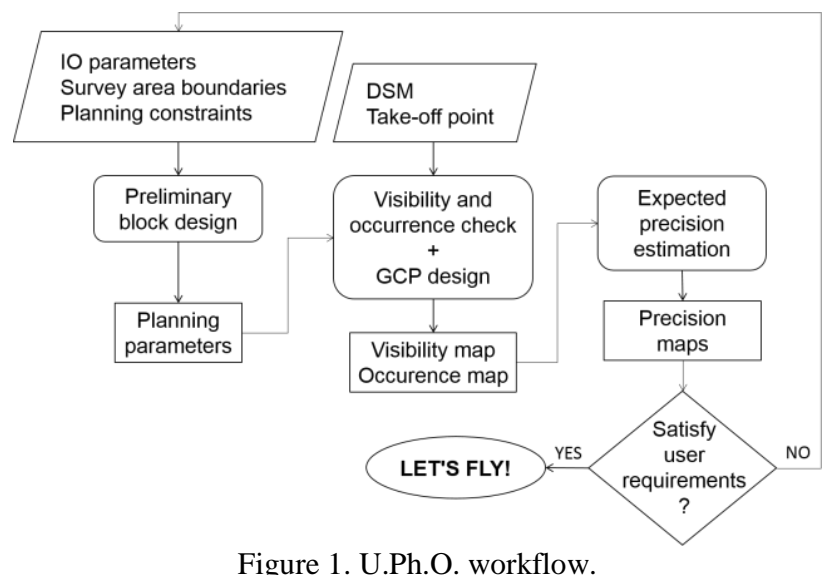

The indication on the achievable precisions can be useful to modify the preliminary survey design, with particular attention to the speed and ease of execution, respecting the metric quality.

Moreover, U.Ph.O. could be particularly useful in emergencies scenarios, where it is important to maintain the correct balance between accuracy and safety of operators, which is strictly related to the distance from the object. It allows a safe survey in areas that otherwise would be inaccessible and, at the same time, it permits to guarantee the respect of metrical precision of the results, in order to document the objects of the survey with an adequate resolution and accuracy, also for conservation and preservation purposes of cultural heritage.

Further details on the rigorous approach could be found in Passoni et al. (2018). 


\section{MAGO WORKFLOW}

MAGO (Adaptive Mesh for Orthophoto Generation) is designed to generate high-resolution orthophoto building a step-by-step adaptive mesh, so to avoid the approximation and loss of definition typically introduced by the mesh reconstruction phase. It also allows to produce a DSM of the object by using several pre-set criteria. The MAGO main operating principles are below summarized.

The required inputs are:

1. the point cloud representing the object;

2. the image and its IO and EO parameters;

3. the orthophoto projection plane;

4. the orthophoto resolution, chosen on the basis of the image GSD;

5. the orthophoto boundaries (optional);

6. the downscaling factor for the original image, to speed-up the procedure and to match the orthophoto resolution.

A regular grid, with resolution in the order of the point cloud spacing, is created to collect the original positions of the input points, to easily recover the whole point cloud information when needed.

Then, the orthophoto is obtained via an iterative process which searches for the plane where the collinearity rays and the point cloud intersect, avoiding any further simplification, re-sample or approximation of the point cloud. At the beginning of the iterative process, the collinearity equations (1) are used to compute the point coordinates $X_{P}$ and $Y_{P}$, having assumed $Z_{P}$ equal to the maximum point cloud height $\left(Z_{M A X}\right)$. The image coordinates $(x, y)$, the IO parameters (focal length $c$, and principal point coordinates, $x_{0}, y_{0}$ ), and the EO parameters (camera positions $\underline{X}_{0}=\left(X_{0}, Y_{0}, Z_{0}\right)$ and $r_{i j}$ components of the rotation matrix $\boldsymbol{R})$ are known.

$$
\begin{aligned}
& x=x_{0}-c \frac{r_{11}\left(X_{P}-X_{0}\right)+r_{12}\left(Y_{P}-Y_{0}\right)+r_{13}\left(Z_{P}-Z_{0}\right)}{r_{31}\left(X_{P}-X_{0}\right)+r_{32}\left(Y_{P}-Y_{0}\right)+r_{33}\left(Z_{P}-Z_{0}\right)} \\
& y=y_{0}-c \frac{r_{21}\left(X_{P}-X_{0}\right)+r_{22}\left(Y_{P}-Y_{0}\right)+r_{23}\left(Z_{P}-Z_{0}\right)}{r_{31}\left(X_{P}-X_{0}\right)+r_{32}\left(Y_{P}-Y_{0}\right)+r_{33}\left(Z_{P}-Z_{0}\right)}
\end{aligned}
$$

The so-obtained $\underline{X}_{P}$ coordinates match a cell of the grid and its corresponding pixel on the orthophoto plane.

If at least one point falls inside the volume defined by the cell dimensions and a threshold fixed on the basis of the point cloud spacing, the correspondence is searched. Thus, a criterion based on the distance between the candidate point and the collinearity ray is introduced. The correspondence is immediately confirmed if the distance is less than a threshold (perfect correspondence). Otherwise, if the threshold is exceeded, the algorithm searches two other points to build a triangle, within the points falling in the individuated cell itself and in the eight neighbouring cells (best-fitting triangle). In the present case, the two candidate points must comply the following requirements:

1. distance criterion: the two individuated points must not be too near or too far from the first one, to avoid an unrealistic description of the surface;

2. non-verticality criterion: the two points must not define a sub-vertical plane;

3. alternation criterion: at least one of the two points must lie on the opposite side of the track of collinearity ray on the orthophoto plane with respect to the first point;

4. area criterion: the individuated triangle must have an area larger than a threshold based on the grid size.

If the previous criteria are met, the intersection between the adaptive triangle and the collinearity ray determines the correspondence between the image and the orthophoto pixels.
In case the first encountered point is not contained in the volume defined by the cell dimensions and the fixed threshold, $Z_{P}$ is decreased of a fixed value depending on point cloud spacing, and the iterative process re-starts. If $Z_{M I N}$ (the minimum height of the points in the point cloud) is reached without having found a matching, the pixel is discarded and the procedure continues analysing a new pixel of the image.

For a more detailed description of criteria and parameters, refer to Gagliolo et al. (2019).

\section{APPLICATION TO THE CASE STUDIES}

A simulation has been carried out considering as case studies the Civic Tower of Norcia and the San Salvatore Church of Campi di Norcia (Italy). The buildings were strongly damaged by the earthquakes that took place in Central Italy in August and October 2016. The operations to provide the securing of the sites were promptly arranged in order to allow the restoring and the retrofitting of the structures and to save the works of art located inside them.

\subsection{Civic Tower (Norcia, Italy)}

The Civic Tower stands out in San Benedetto Square, the core of the historical centre of Norcia. The structure is surrounded by other historical buildings, such as the Basilica of San Benedetto and the Co-Cathedral of Santa Maria Argentea, and private houses. Thus, the examined area is densely built-up, with possible occlusions and limitations for the flight.

Figure 2 depicts the Civic Tower and the façade of San Benedetto Basilica in the day of the UAV survey.

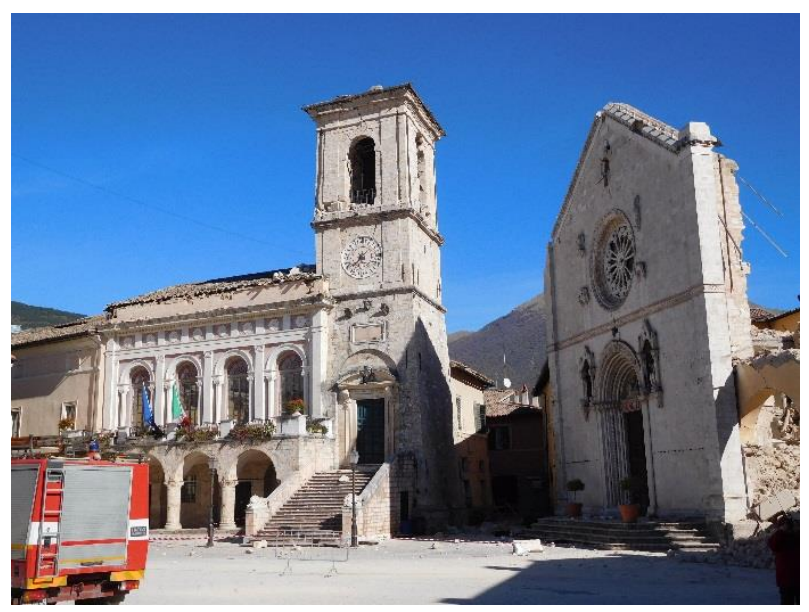

Figure 2. Civic Tower and the façade of San Benedetto Basilica.

Such a complex scenario for a UAV survey was further complicated by the severe safety restrictions applied in case of calamitous events, which strongly limit the direct access to the survey areas and so the flight operations. The aim of the securing operations carried out on the Civic Tower is the disassembly of the damaged structure, in order to avoid an incoherent collapse and then to retrofit and restore it brick-by-brick.

The survey campaign was performed in a single day $\left(3^{\text {rd }}\right.$ November, 2016), followed by one day of photogrammetric postprocessing (thanks to the optimized criteria reported in Gagliolo et al., 2017), which was necessary to design the reinforcing structures, realized on $5^{\text {th }}$ November.

The survey campaign was carried out using a DJI@ Inspire drone with an embedded Zenmuse X3 camera. The focal length is 3.61 $\mathrm{mm}$, while the sensor size is $4000 \times 3000$ pixels with a pixel dimension of $1.56 \mu \mathrm{m}$. The flight was performed considering a 
nadiral attitude and a relative height of about $50 \mathrm{~m}$, which leads to a GSD of $22 \mathrm{~mm}$. A support survey was performed using a GNSS receiver in order to acquire the GCPs coordinates.

\subsection{San Salvatore Church (Campi di Norcia, Italy)}

San Salvatore Church was a small structure located in the countryside of Campi di Norcia. It was surrounded by a wide field, a parking area and it is adjacent to a cemetery.

The aim of the survey was a 3D description of the area, in particular of the church, where the inspection of the interior was important to document the presence of works of art and to design their recovery. The securing operations were particularly devoted to guarantee the safety during the removal of the works of art, and were not addressed to the conservation of the building, because of its highly compromised state, as shown in Figure 3.

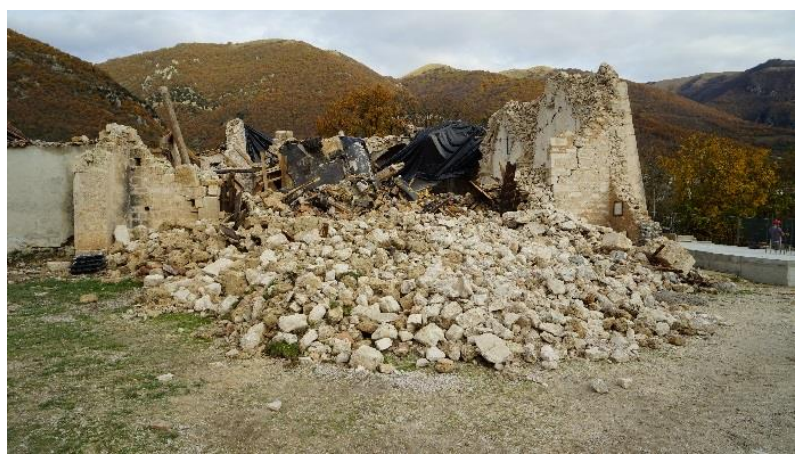

Figure 3. San Salvatore Church ruins.

The survey campaign was performed in a single day $\left(23^{\text {rd }}\right.$ November, 2016), using a DJIC Inspire drone with an embedded Zenmuse X5 camera. The focal length is $15 \mathrm{~mm}$, while the sensor size is $4608 \times 3456$ pixels with a pixel dimension of $3.76 \mu \mathrm{m}$.

The flight was performed considering a nadiral attitude and a relative height of about $50 \mathrm{~m}$, which leads to a GSD of $13 \mathrm{~mm}$. As already mentioned for the Civic Tower, a GNSS survey was performed to acquire the GCPs coordinates.

\subsection{Elaboration settings}

For both the analysed case studies, the U.Ph.O. planning has been carried out with the input parameters listed in Table 1.

\begin{tabular}{|c|c|c|}
\hline \multicolumn{2}{|r|}{ Input parameter } & Value \\
\hline \multicolumn{2}{|c|}{ DSM resolution } & $4 \mathrm{~m}$ \\
\hline \multicolumn{2}{|c|}{ Flight altitude } & $50 \mathrm{~m}$ \\
\hline \multicolumn{2}{|c|}{ Shooting geometry } & Nadiral \\
\hline \multicolumn{2}{|c|}{ GCP number } & 4 \\
\hline \multirow{2}{*}{ Overlapping } & Longitudinal & $70 \%$ \\
\hline & Transversal & $50 \%$ \\
\hline \multirow{3}{*}{$\begin{array}{l}\text { Standard } \\
\text { deviations }\end{array}$} & GCPs coordinates & $3 \mathrm{~cm}$ \\
\hline & Shooting points coordinates & $10 \mathrm{~m}$ \\
\hline & Cameras attitudes & $5^{\circ}$ \\
\hline
\end{tabular}

Table 1. U.Ph.O. input parameters for the two case studies.

The DSMs were obtained from the dense point clouds generated by Agisoft PhotoScan $\odot$, using the Rasterize tool by CloudCompare (2019).

The GCPs have been introduced in order to represent the minimum configuration in terms of GCPs number and arrangement, even if more than four have been measured and used in the photogrammetric post-processing. Despite this, such a limited number of GCPs complies with emergency scenarios, as the case studies are.
The longitudinal and transversal overlapping have been set according to the surveys settings.

The standard deviation of GCPs and shooting points coordinates have been set according to the technique used to measure them: a RTK (Real Time Kinematic) GNSS survey and stand-alone GNSS measurements have been carried out for GCPs and shooting points, respectively, which lead to indicative values of $3 \mathrm{~cm}$ and $10 \mathrm{~m}$. Finally, the cameras attitudes standard deviation has been set to $5^{\circ}$, to account for the UAV instability controlled by the navigation system.

The parameters reported in Table 2 have been set for MAGO.

\begin{tabular}{|l|c|c|}
\hline \multicolumn{1}{|c|}{ Input parameter } & $\begin{array}{c}\text { Value } \\
\text { (Civic Tower) }\end{array}$ & $\begin{array}{c}\text { Value } \\
\text { (San Salvatore) }\end{array}$ \\
\hline Orthophoto resolution & $5 \mathrm{~cm}$ & $5 \mathrm{~cm}$ \\
\hline Grid resolution & $20 \mathrm{~cm}$ & $20 \mathrm{~cm}$ \\
\hline Downscaling factor & 1 (original) & 3 \\
\hline GSD & $2.2 \mathrm{~cm}$ & $1.3 \mathrm{~cm}$ \\
\hline
\end{tabular}

Table 2. MAGO input parameters for the two case studies.

The orthophoto resolution has been set to $5 \mathrm{~cm}$, which is a suitable value for the survey purposes and also considers the achievable precisions. Concerning the grid resolution, it has been fixed to a precautionary value of $20 \mathrm{~cm}$ in both the case studies because the input point clouds have a spacing approximately equal to $10 \mathrm{~cm}$. The downscaling factor has been evaluated according to the ratio between the desired orthophoto resolution and the original image one.

\section{RESULTS AND DISCUSSION}

The results of the application of U.Ph.O. and MAGO on the Civic Tower and San Salvatore Church are presented and discussed in the following sections 5.1 and 5.2. In particular U.Ph.O. provided the visibility map, the occurence map and the estimated precisions along three axes, while MAGO allowed to produce a high resolution orthophoto.

The statistics reported in the following sections have been computed out using GRASS GIS, ver. 7.4 .4 (2019). The Figures reporting U.Ph.O. outputs have been produced using QGIS, ver. 3.4 (2019). The coordinates are expressed in ETRF 2000-2008.0 reference frame, with UTM 32 projection.

\subsection{Civic Tower (Norcia, Italy)}

The visibility map suggests that the Civic Tower is almost obstruction-free, while the areas surrounding it result not visible from the planned shooting points.

The values in the occurrence map (Figure 4) correspond to the number of images that see each DSM cell, taking into account the presence of eventual occlusions.

The black dots represent to the resulting shooting points, obtained in the preliminary block design by U.Ph.O.

The average value of occurrence is 4.5 , with a standard deviation of 1.8. The pattern of Figure 4 underlines what already conceivable from the geometry of both the Civic Tower and the shooting points: the cells with higher occurrence number are located in the centre of the survey area, whereas the occurrence number sharply decreases moving outwards. 


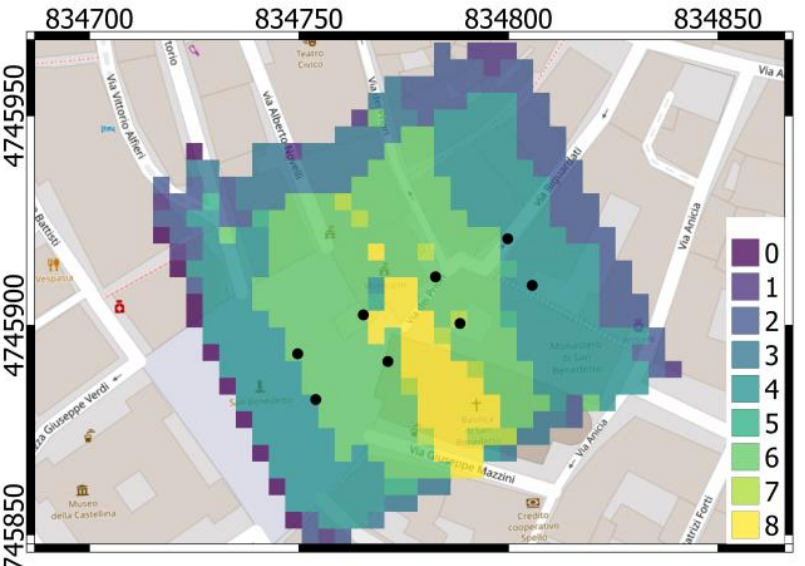

Figure 4. Occurrence map for the Civic Tower.

The occurrence map has been classified, according to the five classes reported in Table 3.

\begin{tabular}{|c|c|c|c|}
\hline Class & $\begin{array}{c}\text { Number } \\
\text { of occurrences }\end{array}$ & Cell count & $\begin{array}{c}\text { Percentage } \\
\text { cover }\end{array}$ \\
\hline 0 & 0,1 & 21 & $3.6 \%$ \\
\hline 1 & 2 & 77 & $13.5 \%$ \\
\hline 2 & 3,4 & 235 & $41.1 \%$ \\
\hline 3 & 5,6 & 188 & $32.9 \%$ \\
\hline 4 & 7,8 & 51 & $8.9 \%$ \\
\hline
\end{tabular}

Table 3. Classification of occurrence map: cell count and percentage cover for the five classes.

Class 1 corresponds to the minimum requirement of occurrences, i.e. two images, to obtain stereo-vision, which is the necessary condition to build a 3D model from images. For the pixels contained in class 0 , it is impossible to reconstruct the point cloud representing the object. For classes 2-4, a 3D model could be successfully reconstructed, also with the possibility of control, thanks to the increasing redundancy with higher class number.

Only a small amount $(3.6 \%)$ of pixels lie in class 0 ; the majority of pixels falls in class 2 , where 3 and 4 number of occurrences are contained, and a significant amount lie in class 3 . Only 11 pixels are not seen ( 0 number of occurrences) and 40 are seen by all the eight shooting points.

In Figure 5Figure 7, the estimated precision along $\mathrm{X}, \mathrm{Y}, \mathrm{Z}$ axes are shown, respectively. The average values of standard deviations are $4.1,4.6$ and $8.5 \mathrm{~cm}$, respectively. As expected, higher values characterize $\sigma_{z}$ with respect to $\sigma_{x}$ and $\sigma_{y}$. For the three maps, the higher values are located along the boundaries of the considered area and on its corners. Observing Figure 7, the lower values of $\sigma_{z}$ for the pixels where the GCPs lie are evident with respect to the surrounding pixels; this is an obvious demonstration of the constraint function of the GCPs, which is even more evident along $\mathrm{Z}$ axis.

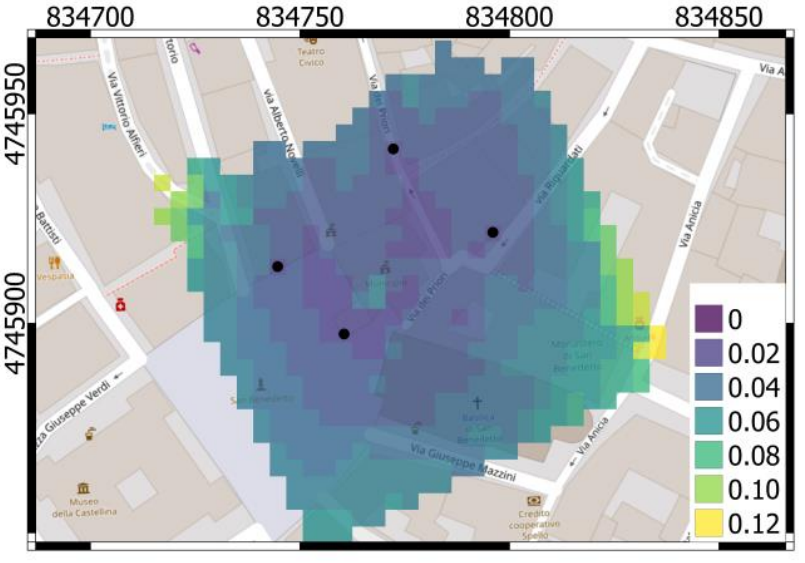

Figure 5. Estimated precisions $[\mathrm{m}]$ along $\mathrm{X}$ axis.

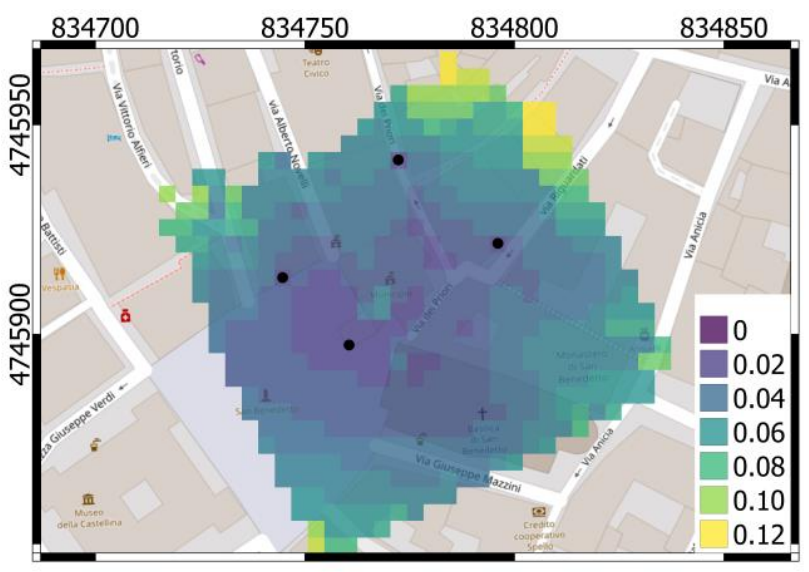

Figure 6. Estimated precisions [m] along $\mathrm{Y}$ axis.

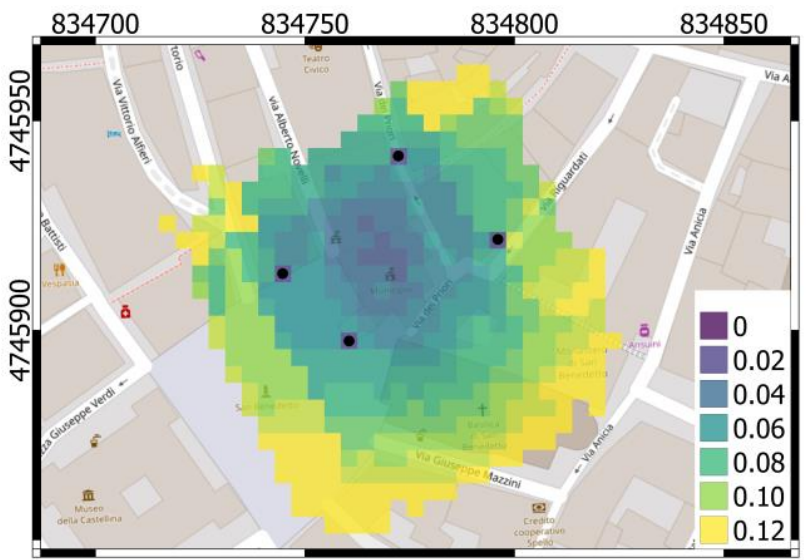

Figure 7. Estimated precisions [m] along $\mathrm{Z}$ axis.

Concerning the post-processing carried out with MAGO, Figure 8 shows the image used for the orthophoto reconstruction (the red rectangle highlights the Civic Tower and its surrounding area depicted in the orthophoto), whereas Figure 9 reports the resulting orthophoto. 


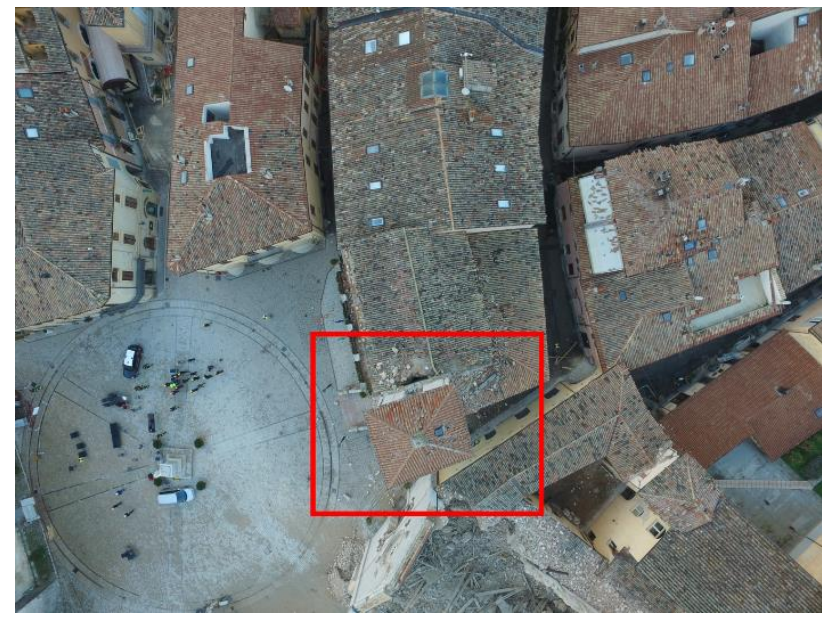

Figure 8. Image to build the orthophoto.

The red rectangle frames the portion depicted in the orthophoto.

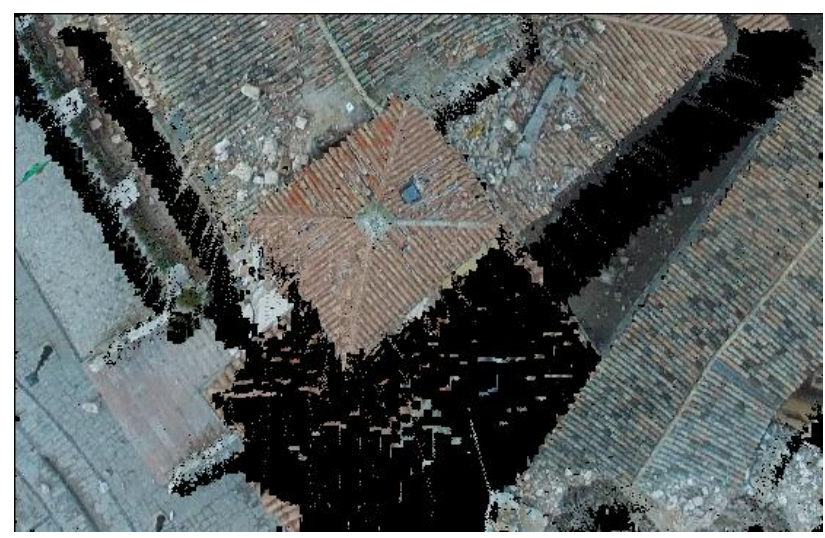

Figure 9. Orthophoto by MAGO.

As evident in Figure 9, some artefacts are present in the black area, which should not be reconstructed because hidden by the Tower roof and the surrounding buildings. Nevertheless, the goodness of orthorectification can be appreciated because the building façades are not visible in the orthophoto.

\subsection{San Salvatore Church (Campi di Norcia, Italy)}

The visibility map suggests that the San Salvatore Church is almost totally visible, thanks to the homogeneous heights of its ruins with respect to the ground, which are not likely to produce obstructions.

The values in the occurrence map (Figure 10Figure 4) report the number of images that see each DSM cell, taking into account the presence of eventual occlusions. The black dots correspond to the resulting shooting point, obtained in the preliminary block design by U.Ph.O.

The average value of occurrence is 3.9 , with a standard deviation of 2.0. The pattern of Figure 10 underlines what already mentioned regarding the Civic Tower, where the shooting geometry is similar to the present case: the cells with higher occurrence number are located in the centre of the survey area, whereas the occurrence number decreases moving towards the more external areas. For San Salvatore Church, the reduction of the number of occurrences has a more gradual behaviour than the one of Civic Tower, also due to the different geometry and features of the two structures.

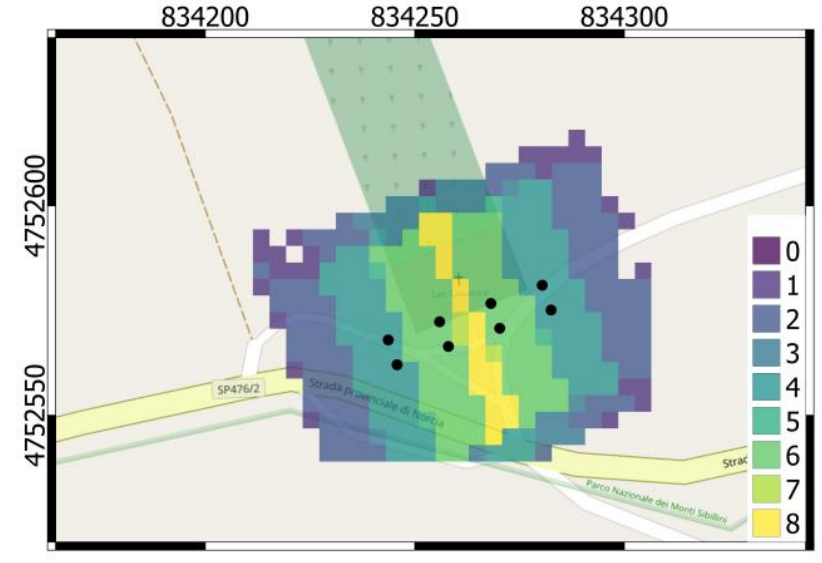

Figure 10. Occurrence map for San Salvatore Church.

The occurrence map has been classified, according to the classes reported in Table 4.

\begin{tabular}{|c|c|c|c|}
\hline Class & $\begin{array}{c}\text { Number } \\
\text { of occurrences }\end{array}$ & Cell count & $\begin{array}{c}\text { Percentage } \\
\text { cover }\end{array}$ \\
\hline 0 & 0,1 & 27 & $7.8 \%$ \\
\hline 1 & 2 & 95 & $27.3 \%$ \\
\hline 2 & 3,4 & 116 & $33.3 \%$ \\
\hline 3 & 5,6 & 87 & $25.0 \%$ \\
\hline 4 & 7,8 & 23 & $6.6 \%$ \\
\hline
\end{tabular}

Table 4. Classification of occurrence map: cell count and percentage cover for the five classes.

Less than $8 \%$ of pixels lie in class 0 ; the majority of the pixels falls in class 2, where 3 and 4 occurrences are contained, and a significant amount lie in class 3 . None pixel is not seen $(0$ number of occurrences) and 20 pixels are seen by all the images.

In Figure 11-Figure 13, the estimated precision along X, Y, Z axes are shown, respectively.

The average values of standard deviations are 2.1,2.1 and $4.6 \mathrm{~cm}$, respectively. As expected, higher values characterize $\sigma_{z}$ with respect to $\sigma_{x}$ and $\sigma_{y}$. For the three maps, the higher values are located along two opposite sides of the considered area. As highlighted in the previous test case, observing Figure 13, the lower values of $\sigma_{z}$ for the pixels where the GCPs lie are evident.

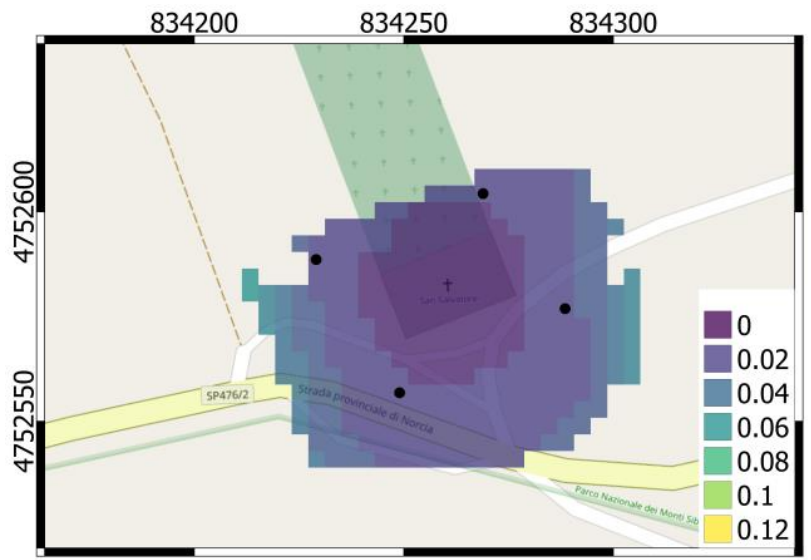

Figure 11. Estimated precisions [m] along $\mathrm{X}$ axis. 


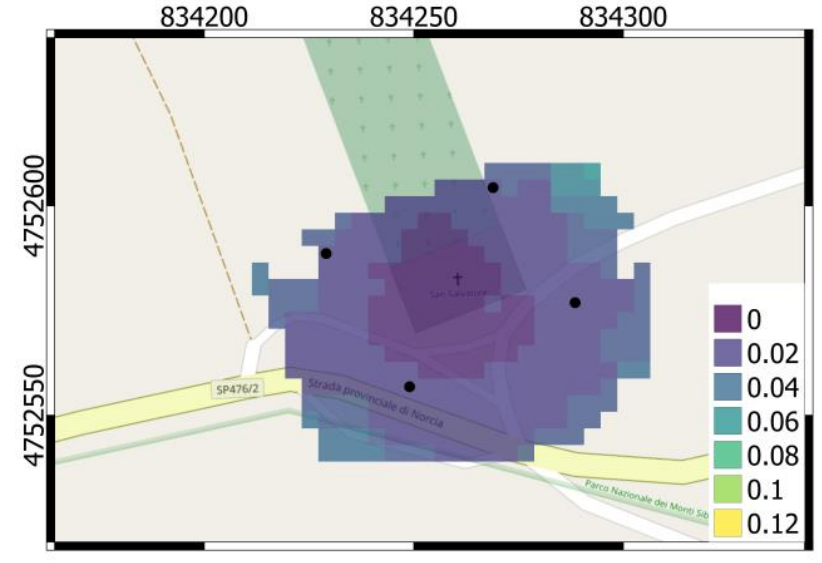

Figure 12. Estimated precisions $[\mathrm{m}]$ along $\mathrm{Y}$ axis.

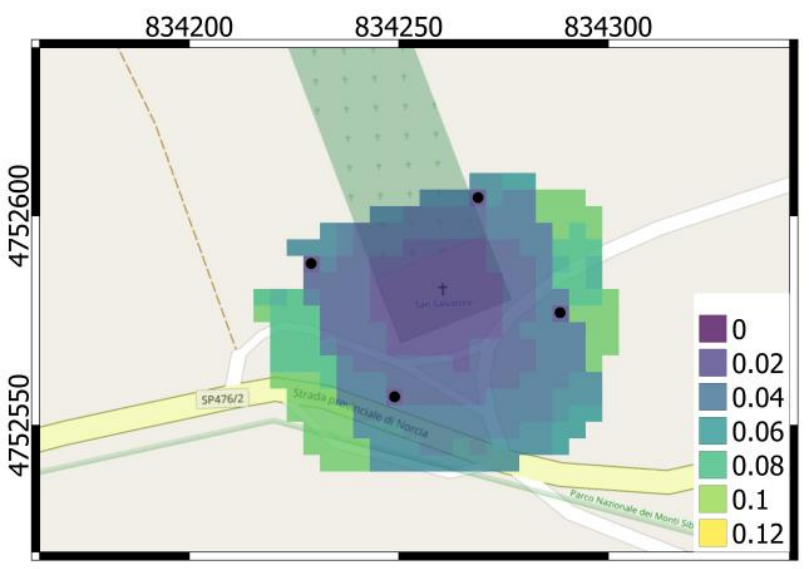

Figure 13. Estimated precisions [m] along $\mathrm{Z}$ axis.

Concerning the post-processing carried out with MAGO, Figure 14Figure 8 shows the image used for the orthophoto reconstruction (the red rectangle highlights the portion depicted in the orthophoto), whereas Figure 15 reports the resulting orthophoto.

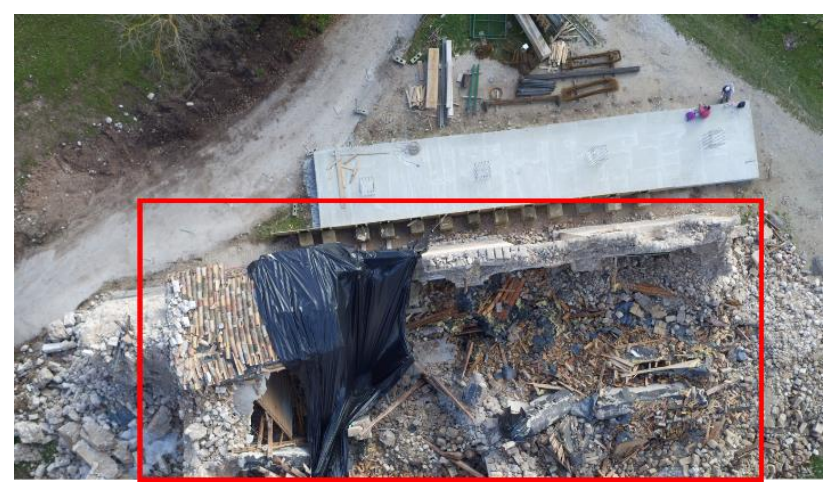

Figure 14. Image to build the orthophoto.

The red rectangle frames the portion depicted in the orthophoto.

As expected, the orthophoto of San Salvatore Church results more complete than the Civic Tower one, thanks to lower height gradient, which does not produce obstructions.

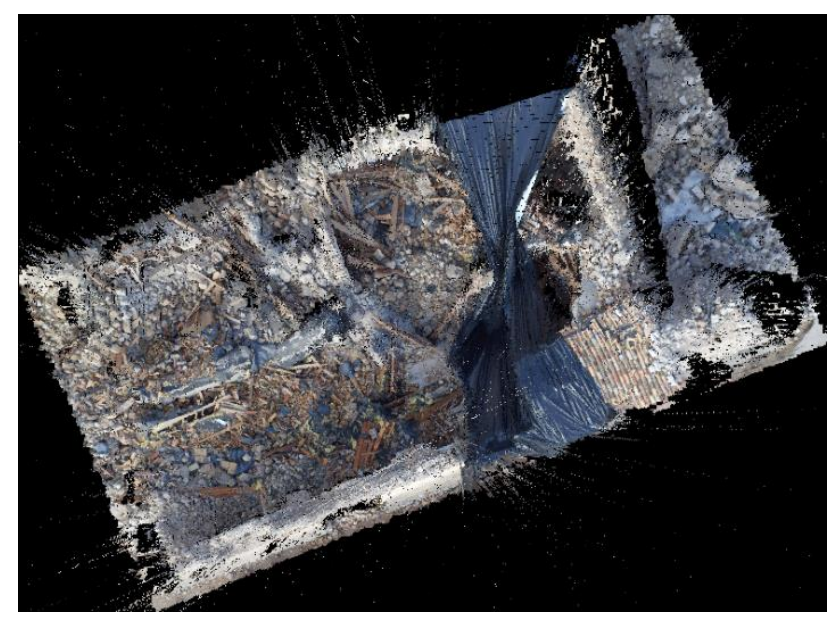

Figure 15. Orthophoto by MAGO.

\section{CONCLUSIONS AND FUTURE PERSPECTIVES}

In the present work, the U.Ph.O (Unmanned Photogrammetric Office) and MAGO (Adaptive Mesh for Orthophoto Generation) tools have been introduced.

The former, implemented in MATLAB ${ }^{\circledR}$ environment, allows the plan an UAV photogrammetric survey campaign accounting for the morphology of the surveyed object and the presence of occlusions in the a-priori estimation of the reachable metric precision. It employs a spatial network optimization by least squares rigorous simulation, considering GCPs distribution, camera parameters knowledge (position and attitude accuracy) and realistic visibility of the object.

MAGO, implemented in C++ environment, allows to generate high-resolution orthophoto building a step-by-step adaptive mesh, avoiding the approximation and loss of definition typically introduced by the building mesh phase. The resulting orthophoto has a resolution of the order of GSD.

U.Ph.O. and MAGO have been applied to two different case studies: a standing-out structure surrounded by buildings (Civic Tower of Norcia) and a collapsed structure in the countryside (San Salvatore Church in Campi di Norcia). The former case more evidently highlights the consequences of the presence of obstructions, which highly influence both the survey planning and restitution. In facts, the occurrence map, the precisions maps and the orthophoto are affected by loss of coverage, precision and visibility, respectively.

On the contrary, San Salvatore Church, which is located in a flat and isolated area, is less impacted by obstructions, thanks to its configuration, which makes the survey planning easier, the achievable precisions higher and the orthophoto more complete than the previous case study.

In conclusion, U.Ph.O. and MAGO are two suitable instrument to optimize the photogrammetric survey planning and restitution, taking into account the realistic morphology of the scene.

The proposed methods are still in development; the aim is to share the tools in the near future through executable files to provide a service for both the scientific and surveyors communities.

\section{ACKNOWLEDGEMENTS}

The authors wish to thank:

- Gter Ltd - Innovation in Geomatics, GNSS and GIS;

- Studio Giampaolo Grosso;

- MiBAC (Italian Ministry of cultural heritage and activities);

- Prof. Stefano Podestà and Yellow Room Engineering. 


\section{REFERENCES}

Achille, C., Adami, A., Chiarini, S., Cremonesi, S., Fassi, F., Fregonese, L., Taffurelli, L., 2015. UAV-based photogrammetry and integrated technologies for architectural applications Methodological strategies for the after-quake survey of vertical structures in Mantua (Italy). Sensors 15(7), pp. 15520-15539.

Adams, S. M., Friedland, C. J., 2011. A survey of Unmanned Aerial Vehicle (UAV) usage for imagery collection in disaster research and management. Proceedings of the Ninth International Workshop on Remote Sensing for Disaster Response, 15-16 September, Stanford, CA, USA.

Agisoft PhotoScan® , 2019, http://www.agisoft.com/ (access on $25^{\text {th }}$ March 2019)

Ballarin, M., Buttolo, V., Guerra, F., Vernier, P., 2013. Integrated surveying techniques for sensitive areas: San Felice sul Panaro. ISPRS Annals of the Photogrammetry, Remote Sensing and Spatial Information Sciences 5, W1.

CloudCompare, 2019, http://www.cloudcompare.org/ (access on $25^{\text {th }}$ March 2019)

Colomina, I., Molina, P., 2014. Unmanned Aerial Systems for Photogrammetry and Remote Sensing: a review. ISPRS Journal of Photogrammetry and Remote Sensing, Vol. 92, pp. 79-97.

Cryderman, C., Mah, S.B., Shufletoski, A., 2014. Evaluation of UAV Photogrammetric Accuracy for Mapping and Earthworks Computations. GEOMATICA, 68(4), pp. 309-317.

Everaerts, J., 2008. The use of Unmanned Aerial Vehicles (UAVs) for Remote Sensing and mapping. ISPRS Archives, XXI ISPRS Congress, 3-11 July, Beijing, China, Vol. 37/B1, pp. 1187-1192.

Gagliolo, S., Fagandini, R., Federici, B., Ferrando, I., Passoni, D., Pagliari, D., Pinto, L., Sguerso, D., 2017. Use of UAS for the conservation of historical buildings in case of emergencies. International Archives of the Photogrammetry, Remote Sensing and Spatial Information Sciences, Vol. XLII-5/W1, p. 81-88.

Gagliolo, S., Federici, B., Ferrando, I., Sguerso, D., 2019. MAGO: a new approach for orthophotos production based on adaptive mesh reconstruction. International Archives of the Photogrammetry, Remote Sensing and Spatial Information Sciences (in print).

GRASS GIS ver. 7.4.4, 2019, https://grass.osgeo.org/ (access on $25^{\text {th }}$ March 2019)

Grün, A., 1980. Precision and reliability aspects in Close-Range photogrammetry. XIV Congress of ISP, Comm. V., Hamburg.

James, M.R., Robson, S., Smith, M.W., 2017. 3-D uncertaintybased topographic change detection with structure-from-motion photogrammetry: precision maps for ground control and directly georeferenced surveys. Earth Surface Processes and Landforms, 42(12), pp. 1769-1788.

Meyer, D., Hess, M., Lo, E., Wittich, C. E., Hutchinson, T. C., Kuester, F., 2015. UAV-based post disaster assessment of cultural heritage sites following the 2014 South Napa Earthquake. Digital Heritage, Vol. 2, pp. 421-424.
QGIS ver 3.4, 2019, https://www.qgis.org (access on 25 $5^{\text {th }}$ March 2019)

Passoni, D., Federici, B., Ferrando, I., Gagliolo, S., Sguerso, D., 2018. The estimation of precisions in the planning of UAS photogrammetric surveys, Int. Arch. Photogramm. Remote Sens. Spatial Inf. Sci., XLII-2, pp. 837-843.

Remondino, F., Barazzetti, L., Nex, F., Scaioni, M., Sarazzi, D., 2011. UAV photogrammetry for mapping and 3D modeling Current status and future perspectives. Int. Arch. Photogramm. Remote Sens. Spatial Inf. Sci., XXXVIII-1/C22, pp. 1-7.

Sona, G., Passoni, D., Pinto, L., Pagliari, D., Masseroni, D., Ortuani, B., Facchia, A., 2016. UAV multispectral survey to map soil and crop for precision farming applications. Int. Arch. Photogramm. Remote Sens. Spatial Inf. Sci., XLI-B1, pp. 10231029.

Torlegård, K., 1980. On accuracy improvement in Close-Range photogrammetry, Proceedings of the Industrial and Engineering Survey Conference, London. 